\title{
Pensar para Programar: Projeto de Ensino no Curso Técnico em Informática
}

\author{
Wagner dos Santos Marques, Paulo Silas Severo de Souza, \\ Jaline Gonçalves Mombach \\ ${ }^{1}$ Instituto Federal Farroupilha (IF Farroupilha) \\ Campus Alegrete - RS - Brasil \\ \{wagner.marques, paulo.souza\}@email.com, \\ jaline.mombacheiffarroupilha.edu.br
}

\begin{abstract}
This paper presents a report of the experiences obtained during a teaching project developed with first-year students of an Informatic Technician course. It was used concepts of Unplugged Computing and Problem-Based Learning in order to instigate the development of skills related to the Computational Thinking on the students. The results showed that such methodology was successful, contributing to the development of student's cognitive skills, such as critical thinking and problem-solving.
\end{abstract}

Resumo. Este artigo apresenta um relato das experiências obtidas durante um projeto de ensino realizado com alunos do primeiro ano de um curso de Técnico em Informática. Foram usados conceitos de computação desplugada e aprendizagem baseada em problemas para instigar o desenvolvimento de habilidades relacionadas ao pensamento computacional nos alunos. Os resultados mostraram que tal metodologia foi bem-sucedida, contribuindo para o desenvolvimento de habilidades cognitivas dos alunos, como pensamento crítico e resolução de problemas.

\section{Introdução}

Um dos principais problemas enfrentados pelas instituições de ensino superior e técnico é a alta evasão dos alunos em cursos da área de Ciência da Computação. Em 2014, surgiram cerca de 78 mil novas vagas no setor de TI (Tecnologia da Informação), e destas, apenas 33 mil foram preenchidas, o que ilustra a carência de profissionais nesta área, causada por problemas como a evasão escolar (GALDINO; NETO; COSTA, 2015).

A evasão escolar em cursos de computação pode ocorrer por diversos motivos, entre eles o fato de muitos alunos apresentarem dificuldades em disciplinas que exigem raciocínio lógico, como algoritmos e programação. Além disso, professores possuem dificuldades ao apresentar atividades que exercitam o raciocínio lógico, pois nem sempre dispõem de material didático apropriado para este fim (GALDINO; NETO; COSTA, 2015).

Nesse sentido, vários estudos propõem práticas e ferramentas que estimulam o desenvolvimento de habilidades como o raciocínio lógico dos estudantes. Dentre os temas abordados, destaca-se o pensamento computacional, que pode ser entendido como 
a aplicação de conceitos de Ciência da Computação e de ferramentas $\operatorname{cognitivas}^{1}$ na resolução de problemas (WING, 2006). O objetivo de incluir atividades que desenvolvem o pensamento computacional nos alunos é proporcionar uma aprendizagem mais efetiva em disciplinas relacionadas à computação (FRANÇA; AMARAL, 2012).

Assim, com o intuito de diminuir a evasão e facilitar o aprendizado dos alunos do curso técnico em Informática do Instituto Farroupilha Campus Alegrete, foi desenvolvido o projeto "Pensar para Programar", que tem como objetivo trabalhar o pensamento computacional dos alunos por meio de ferramentas e materiais didáticos destinados ao exercício de lógica. Assim, este trabalho apresenta um relato das experiências obtidas durante o desenvolvimento deste projeto de ensino, visando orientar e incentivar ações semelhantes em outras instituições.

O restante deste artigo está organizado como segue. Na Seção 2 são apresentados trabalhos relacionados ao projeto desenvolvido; na Seção 3 discorre-se sobre o referencial teórico utilizado. A Seção 4 é reservada ao relato das experiências obtidas no decorrer do projeto, e na Seção 5 são feitas as considerações finais.

\section{Trabalhos Relacionados}

Vários estudos apresentam as vantagens do incentivo ao pensamento computacional. Oliveira e Araujo (2016) investigaram as habilidades relacionadas ao pensamento computacional que podem ser desenvolvidas por meio de atividades de robótica. Após a realização de uma revisão da literatura, os autores realizaram uma oficina de robótica a fim de explorar tais habilidades. Como avaliação da proposta, foram realizados testes e questionários. Os resultados foram positivos, mostrando que após a oficina os alunos foram capazes de resolver problemas de forma mais eficaz.

Souza, Rodrigues e Andrade (2016) promoveram um curso de robótica para turmas do ensino médio. $\mathrm{O}$ intuito era diminuir a deficiência na formação docente e discente em competências do pensamento computacional. A proposta foi avaliada quantitativamente por meio da avaliação do desempenho de 729 alunos. Os autores apontam que o desempenho dos alunos foi significativamente aprimorado após sua participação no curso. Além disso, os autores enfatizam que a inserção do pensamento computacional melhorou o desempenho dos alunos nas oficinas de robótica tradicionalmente ofertadas.

Rodrigues et al. (2015) empreenderam um estudo quantitativo para analisar o impacto do pensamento computacional no desempenho de estudantes da educação básica. $\mathrm{O}$ projeto envolveu 103 estudantes do Estado da Paraíba, divididos em dois grupos de amostra, sendo que um dos grupos já tinha experiência em programação. Os resultados obtidos indicaram que a prática de programação pode aprimorar e influenciar no desempenho de alunos em diferentes contextos através do pensamento computacional.

Pinho et al. (2016) descrevem os resultados da aplicação da ferramenta "Caça ao Tesouro", que aborda conceitos de busca de dados desenvolvidos no âmbito do pensamento computacional. Foi proposto um jogo digital como ferramenta para a execução das tarefas. Após a avaliação da proposta, os resultados da abordagem utilizada foram comparados aos resultados obtidos através de uma aplicação do método clássico. Os dados

\footnotetext{
${ }^{1}$ Ferramentas cognitivas são elementos tecnológicos que podem ser usados para instigar o pensamento crítico dos alunos acerca do que estes estão estudando. (JONASSEN; CARR; YUEH, 1998).
} 
preliminares indicam que a abordagem é satisfatória.

Falcão e Barbosa (2015) apontam a falta de métodos para realização de avaliações de qualidade de jogos digitais que envolvem a lógica de programação e que visam o desenvolvimento do pensamento computacional. Nesse sentido, através do estudo de caso, os autores apresentam uma análise dos aspectos pedagógicos e técnicos que podem ser usados no processo de interação de crianças com jogos desenvolvidos para tal propósito. Ao final de seu estudo, os autores enfatizam que os aspectos analisados possuem potencial para se tornarem parâmetros de um método de avaliação formal.

Zanetti, Borges e Ricarte (2016) realizaram uma revisão sistemática da literatura dos trabalhos relacionados ao pensamento computacional. Foram analisados 16 artigos a fim de apresentar uma visão da área de pesquisa e definir quais as linhas de atuação dos pesquisadores no Brasil. Os resultados indicam que as práticas mais abordadas por estudos que visam disseminar o pensamento computacional são as Linguagens de programação visual, computação desplugada e jogos digitais.

O diferencial deste trabalho em relação aos demais contidos na literatura é o relato de experiência e os resultados obtidos após a adoção de jogos de tabuleiro e de lógica matemática com o intuito de melhorar o pensamento computacional dos estudantes e dessa forma facilitar o aprendizado destes nas disciplinas de programação nos cursos de computação.

\section{Referencial Teórico}

Nesta Seção são descritos os principais conceitos e características da metodologia de ensino adotada.

\subsection{Pensamento Computacional e Resolução de Problemas}

A metodologia baseada no pensamento computacional usa conceitos de Ciência da Computação para aprimorar as habilidades criativas e de resolução de problemas dos alunos. A aplicação de atividades que exploram o pensamento computacional facilita a comunicação entre alunos e professores, permitindo que ambos falem a mesma linguagem durante a discussão de problemas e a análise de possíveis soluções (FURBER et al., 2012).

O trabalho em equipe é incentivado em práticas que envolvem o pensamento computacional, pois permite que os alunos discutam durante a resolução dos problemas, aprimorando habilidades como reflexão e pensamento crítico. Neste sentido, percebe-se que abordagens como a aprendizagem baseada em problemas, que estimulam o trabalho em equipe, podem ser usadas para estimular o desenvolvimento do pensamento computacional.

A aprendizagem baseada em problemas (ABP) é uma metodologia educacional desenvolvida na McMaster University's Faculty of Health Sciences que usa a resolução colaborativa de problemas para aumentar a eficácia do processo de aprendizagem. Nesta metodologia, o professor apresenta um problema prático, e os discentes reúnem-se em grupos de estudo para tentar resolvê-lo. A ABP é considerada uma metodologia centrada nos alunos, pois estes devem assumir a responsabilidade por seu próprio aprendizado, levantando hipóteses de forma colaborativa e identificando quais conteúdos são necessários 
para solucionar o problema. Após completarem o desafio, a ABP sugere que todos alunos se reúnam com seus professores, para discutir sobre as experiências obtidas (BARROWS, 1996).

\subsection{Computação Desplugada}

A computação desplugada é uma técnica que consiste em ensinar os fundamentos da computação através de atividades lúdicas que não fazem uso de um computador, favorecendo um ambiente sem distrações e detalhes técnicos em demasia. Tal técnica é composta por diversas atividades e pode ser aplicada à pessoas de diferentes idades, com diversos níveis de escolaridade e com diferentes conhecimentos e experiências (VIEIRA; PASSOS; BARRETO, 2013).

Além disso, é possível a realização destas práticas pedagógicas em diferentes estruturas escolares de forma simples e interativa, pois tal metodologia não necessita de componentes de hardware, nem de softwares para ser aplicada (COSTA et al., 2012).

\subsection{Práticas Colaborativas}

As práticas colaborativas permitem que problemas propostos possam ser resolvidos de maneira colaborativa, onde os educandos podem dividir as tarefas e analisar quais as soluções mais eficazes para realizá-los. Ressalta-se ainda que esta abordagem é benéfica aos alunos, pois torna possível a troca de experiências e o desenvolvimento de habilidades sociais e trabalho em equipe (RAMOS, 2011).

Além disso, o trabalho colaborativo permite que os alunos complementem seus conhecimentos e tornem-se mais criativos através da troca de conhecimentos e ideias. Também, a tecnologia destaca-se por ampliar práticas colaborativas através de computadores (GEROSA; FUKS; LUCENA, 2003).

\section{Relato de Experiência}

Esta seção descreve detalhadamente as atividades realizadas durante o projeto, explicando o contexto no qual as atividades foram aplicadas, o perfil dos alunos, e também as experiências obtidas.

\subsection{Contexto e Metodologia}

O projeto "Pensar para Programar" foi realizado no Instituto Federal de Educação, Ciência e Tecnologia Farroupilha (IFFarroupilha) - Campus Alegrete, com aproximadamente 27 alunos pertencentes à turma do primeiro ano do curso técnico em Informática integrado ao ensino médio. A equipe executora constituiu-se de uma professora coordenadora e de dois acadêmicos do curso de Análise e Desenvolvimento de Sistemas.

O projeto foi realizado entre os meses de agosto e dezembro de 2016, em encontros semanais com duração de duas horas. Cada encontro foi dividido em duas etapas de uma hora. Na primeira etapa, eram aplicados jogos de lógica e de incentivo ao pensamento computacional. Em seguida, na segunda etapa do encontro, realizavam-se exercícios de algoritmos relacionados ao conteúdo ministrado na disciplina de "Programação I". A fim de auxiliar a primeira parte do encontro, utilizaram-se algumas ferramentas e materiais: 
- Lobogames ${ }^{2}$ : projeto mantido pela Universidade Federal do Rio Grande do Sul (UFRGS), que promove o uso de jogos lógicos de tabuleiro para facilitar o desenvolvimento do raciocínio lógico. O projeto disponibiliza diversos modelos de tabuleiros para impressão e uso livre em atividades de ensino.

- Blockly ${ }^{3}$ : sistema criado pela Google destinado ao ensino de programação. Tal ferramenta oferece diversas atividades educativas e permite a programação em blocos que representam comandos, ideal para entendimento inicial da lógica de programação.

- Jogos Boole ${ }^{4}$ : revistas com diferentes níveis de desafios de raciocínio lógico por meio de histórias baseadas em estruturas lógico-matemáticas, em forma de enigmas e problemas. Todas as histórias são resolvidas com um jogo de cartas disponibilizadas junto aos livros.

- Problemas de Lógica: histórias similares ao "Teste de Einstein", desafio lógico popular em formato de tabela, disponibilizadas no portal Racha $\mathrm{Cuca}^{5}$, além de modelos de quebra-cabeça em madeira.

Além disso, os monitores do projeto acompanharam as aulas de "Programação I", ministradas pela coordenadora do projeto, com o intuito de acompanhar os alunos e auxiliar a docente no processo de ensino da disciplina.

\subsection{Aula Experimental}

Foi realizada uma aula experimental junto à disciplina de "Programação I", ministrada pela coordenadora do projeto, a fim de verificar se as ações planejadas seriam aceitas e despertariam o interesse dos alunos. Neste encontro, foi adotado o projeto Lobogames em duas modalidades: Tabuleiro e Jogo Vivo. Na modalidade tabuleiro, o jogo é apresentado em sua forma tradicional, com um tabuleiro sobre a mesa e apenas dois jogadores (Figura 1(a)). Já no Jogo Vivo (Figura 1(b)), os jogadores tornam-se as peças do tabuleiro. Esta modalidade é centrada no desenvolvimento do trabalho em equipe dos alunos.

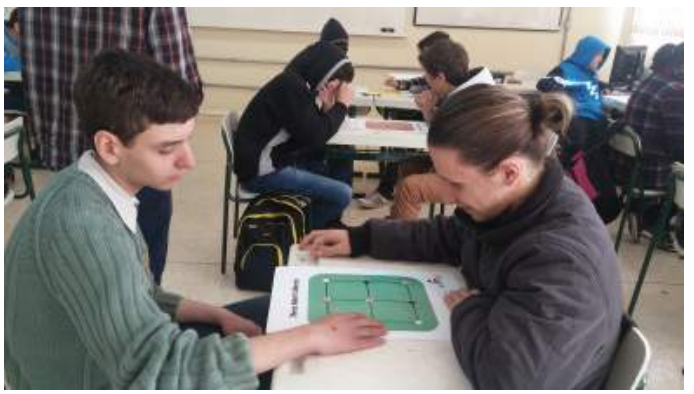

(a) Tabuleiro

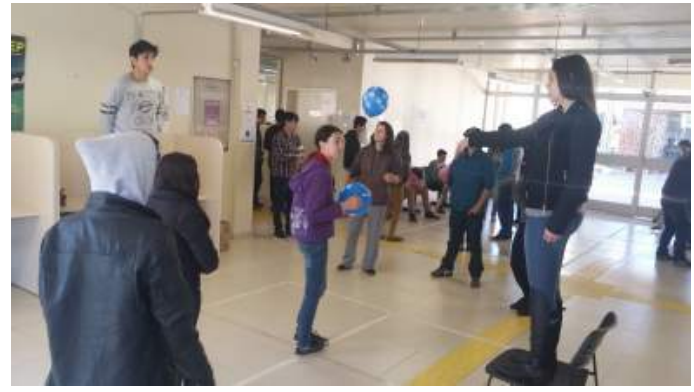

(b) Jogo Vivo

Figura 1. Registro da realização da aula experimental.

Os alunos mostraram entusiasmo e curiosidade em relação ao material elaborado. Ao final do encontro, os educandos foram reunidos e questionados a respeito do interesse

\footnotetext{
${ }^{2}$ Lobogames. Disponível em: <http://www.inf.ufrgs.br/lobogames $>$.

${ }^{3}$ Blockly. Disponível em: $<$ https://developers.google.com/blockly $>$.

${ }^{4}$ Jogos Boole. Disponível em: <http://www.jogosboole.com.br $>$.

${ }^{5}$ Racha Cuca. Disponível em: <https://rachacuca.com.br/logica/problemas $>$.
} 
em participar do projeto, momento em que expressaram aceitação à metodologia proposta e interesse em participar do projeto. Nesse sentido, a abordagem planejada mostrou-se satisfatória.

\subsection{Encontros}

Inicialmente, o projeto seria ofertado apenas para os alunos que haviam obtido notas abaixo da média no primeiro semestre do corrente ano. Todavia, no primeiro encontro após a aula experimental, os demais alunos com notas acima da média solicitaram a participação no projeto junto aos demais colegas, o que foi permitido.

Nos primeiros encontros, os discentes eram instruídos a realizar desafios de lógica propostos. Inicialmente alguns alunos destacaram-se dos demais, apresentando maior facilidade de resolução de problemas lógico-matemáticos. Além disso, notou-se colaboração entre os envolvidos durante os encontros, e uma melhora significativa no pensamento computacional dos estudantes.

A cada encontro, a equipe executora do projeto adotava uma das ferramentas citadas anteriormente, aumentando o nível de dificuldade ao longo das aulas. Nesse sentido, os encontros não se tornaram repetitivos, evitando que os alunos perdessem o interesse por tais atividades.

Ao decorrer das atividades, foram identificados os alunos que apresentavam maiores dificuldades e que necessitavam de atenção singular. Nesse sentido, os executores passaram a adaptar as atividades para estes discentes a fim de auxiliá-los no processo de aprendizagem, como por exemplo, um aluno com déficit de atenção que apresentava dificuldades na realização de exercícios de programação em sala de aula mas desempenho acima da média nos jogos de lógica do projeto.

\subsection{Feira de Ciências}

A fim de selecionar alguns alunos para apresentar o projeto junto à Feira de Ciências, evento promovido pelo IFFarroupilha na comunidade alegretense, realizou-se uma competição de desafios lógicos. Dois educandos teriam a oportunidade de apresentar o projeto à comunidade local durante o referido evento. Logo, os estudantes foram divididos em duplas e um desafio dos jogos Boole foi proposto, e a equipe que resolvesse o problema de lógica primeiro e de forma correta, venceria.

Após o fim do encontro, os desafios foram corrigidos e o ranqueamento das duplas foi realizado. Em seguida, as três duplas com melhor colocação foram questionadas sobre o interesse de participar da Feira, momento em que todos esboçaram interesse em representar os colegas em tal evento. Nesse sentido, os seis alunos foram autorizados a realizar a apresentação com o auxílio dos monitores do projeto. A Figura 2 registra a participação dos alunos na Feira.

O material dos projetos Lobogames e Jogos Boole foram levados ao evento. Os alunos selecionados demonstraram domínio do material, ao passo que instruíram discentes de outras instituições e comunidade em geral a realizar os desafios e os jogos de tabuleiro.

Ficou evidenciado o progresso de alunos que tinham dificuldade de raciocínio lógico, visto que estes mostraram proficiência ao auxiliar os demais participantes da feira durante a realização dos desafios elaborados. 


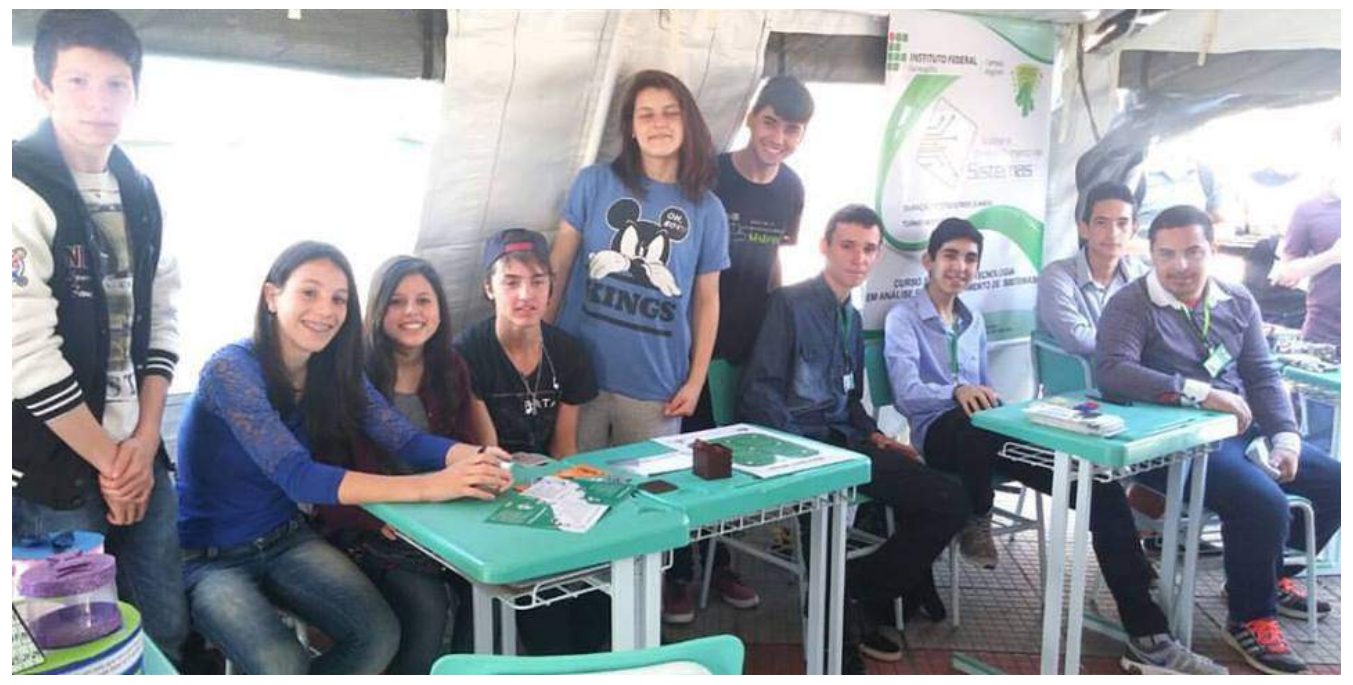

Figura 2. Participação dos alunos na Feira de Ciências

\subsection{Competição de Algoritmos}

Ainda com o objetivo de incentivar o trabalho em equipe e o pensamento computacional, foi promovida uma competição entre os os alunos. Foram desenvolvidos problemas com pontuações diversas de acordo com sua complexidade, que deveriam ser solucionados através de algoritmos. Os alunos foram encaminhados para um ambiente externo ao da sala de aula e poderiam resolver os problemas com auxílio de editores instalados em seus próprios smartphones. Cada equipe podia sortear um problema para resolução e só ganhava novo desafio ao mostrar que o algoritmo desenvolvido solucionava o problema. No quadro 1 são descritos dois problemas, exemplificando a dinâmica.

\section{PONTUAÇÃO: 20 PONTOS}

Wagner cansou de ser bolsista na monitoria e resolveu tentar a sorte na Mega-Sena. Cada jogo é composto por seis números, de 1 a 60. Se o jogador escolher "Surpresinha", o sistema gera os números automaticamente. Por isso, faça um algoritmo que gere dois jogos "surpresinha" para o Wagner. Use estruturas de repetição.

\section{PONTUAÇÃO: 25 PONTOS}

No pomar de Dona Mariquinha tem um pé de laranjeira e um pé de bergamoteira. Consideramos que hipoteticamente, a bergamoteira cresce $20 \mathrm{~cm}$ por ano e a laranjeira $30 \mathrm{~cm}$ por ano, faça um algoritmo que determine quantos anos demorará para que a laranjeira seja maior que a bergamoteira. Atualmente a bergamoteira tem $100 \mathrm{~cm}$ e a laranjeira tem $80 \mathrm{~cm}$.

Quadro 1: Exemplo de problemas elaborados para a competição.

Os discentes foram divididos em equipes de cinco integrantes e a equipe com maior pontuação ao final da atividade ou que resolvessem todos os problemas primeiro, ganharia a competição e receberia uma premiação. Ao final, após a contagem dos pontos, definiu-se a equipe ganhadora. Surpreendentemente, a equipe vencedora era constituída por alunos que não haviam alcançado média na disciplina de "Programação I" no semestre 
anterior, o que mostrou o progresso dos educandos e a contribuição do projeto no processo de aprendizado dos participantes.

\section{Considerações Finais}

Este trabalho relata as experiências obtidas através do projeto "Pensar para Programar", que teve como objetivo reduzir a evasão e melhorar o aproveitamento na disciplina Programação I do curso Técnico em Informática no Instituto Federal Farroupilha - Campus Alegrete. O projeto foi constituído de atividades provenientes da computação desplugada e da aprendizagem baseada em problemas, a fim de melhorar o pensamento computacional dos alunos participantes.

No decorrer do projeto, foi possível perceber o desenvolvimento dos alunos em relação à habilidade de resolução de problemas. Além disso, alguns discentes que tinham dificuldades na disciplina de programação destacaram-se durante as atividades do projeto. Também percebeu-se uma grande colaboração entre os educandos, o que evidenciou o aumento da habilidade de trabalho em equipe dos participantes gerado pela metodologia adotada.

No entanto, a partir da décima semana o horário das disciplinas precisou ser alterado e o projeto, que ocorria em períodos disponíveis entre aulas regulares no turno da manhã, passou a ocorrer no turno da tarde, inverso ao das aulas. Percebeu-se a diminuição de participantes, visto que alguns já estavam comprometidos em horas de recuperação paralela com outras disciplinas e outros não ficavam na instituição. Todavia, dos alunos ativos, a maioria mostrou-se beneficiada pela metodologia adotada.

A docente da disciplina avaliou o projeto de forma positiva, visto que alguns alunos de fato tiveram melhor rendimento em aula e ao final do ano letivo apenas dois alunos desistiram do curso, mas mesmo assim, não saíram da instituição, apenas ingressaram em outro curso técnico. Destaca-se o caso do aluno com déficit de atenção, pois este apresentava baixa autoestima e dificuldade de entrosamento com os demais colegas, provavelmente pela dificuldade de realização dos exercícios em aula. Porém, conforme avaliação da docente, este aluno demonstrou maior segurança e notou-se progresso principalmente na comunicação com os colegas, visto que ele apresentava facilidade na interação com jogos de tabuleiro e em alguns desafios lógicos.

Quanto à equipe executora, a experiência foi considerada enriquecedora. Como trabalhos futuros, almeja-se a continuidade do projeto com novas turmas contendo práticas pedagógicas relacionadas à computação desplugada.

\section{Referências}

BARROWS, H. S. Problem-based learning in medicine and beyond: A brief overview. New directions for teaching and learning, Wiley Online Library, v. 1996, n. 68, p. 3-12, 1996.

COSTA, T. et al. Trabalhando fundamentos de computação no nível fundamental: experiência de licenciandos em computação da universidade federal da paraíba. In: $X X$ Workshop de Educação em Computação-WEI. Curitiba, PR, Brasil. [S.l.: s.n.], 2012.

FALCÃO, T. P.; BARBOSA, R. "aperta o play!"análise da interação exploratória em um jogo baseado em pensamento computacional. In: Brazilian Symposium on Computers in 
Education (Simpósio Brasileiro de Informática na Educação-SBIE). [S.1.: s.n.], 2015. v. 26, n. 1, p. 419.

FRANÇA, R.; AMARAL, H. Proposta de um jogo eletrônico educativo aplicado ao ensino da lógica de programação. In: CONFERÊNCIA LATINOAMERICANA DE OBJETOS E TECNOLOGIAS DE APRENDIZAGEM. [S.1.: s.n.], 2012. v. 7.

FURBER, S. et al. Shut down or restart? the way forward for computing in uk schools. The Royal Society, London, 2012.

GALDINO, C.; NETO, S.; COSTA, E. Kidcoder: Uma proposta de ensino de programação de forma lúdica. In: Brazilian Symposium on Computers in Education (Simpósio Brasileiro de Informática na Educação-SBIE). [S.1.: s.n.], 2015. v. 26, n. 1, p. 687.

GEROSA, M. A.; FUKS, H.; LUCENA, C. J. P. de. Suporte à percepção em ambientes de aprendizagem colaborativa. Brazilian Journal of Computers in Education, v. 11, n. 2, p. 75-85, 2003.

JONASSEN, D. H.; CARR, C.; YUEH, H.-P. Computers as mindtools for engaging learners in critical thinking. TechTrends, Springer, v. 43, n. 2, p. 24-32, 1998.

OLIVEIRA, E.; ARAUJO, A. L. Pensamento computacional e robótica: Um estudo sobre habilidades desenvolvidas em oficinas de robótica educacional. In: Brazilian Symposium on Computers in Education (Simpósio Brasileiro de Informática na Educação-SBIE). [S.1.: s.n.], 2016. v. 27, n. 1, p. 530.

PINHO, G. et al. Proposta de jogo digital para dispositivos móveis: Desenvolvendo habilidades do pensamento computacional. In: Brazilian Symposium on Computers in Education (Simpósio Brasileiro de Informática na Educação-SBIE). [S.1.: s.n.], 2016. v. 27, n. 1 , p. 100.

RAMOS, D. K. Processos colaborativos mediados pelo computador e as contribuições da teoria da atividade. Brazilian Journal of Computers in Education, v. 18, n. 03, p. 34, 2011.

RODRIGUES, R. da S. et al. Análise dos efeitos do pensamento computacional nas habilidades de estudantes no ensino básico: um estudo sob a perspectiva da programação de computadores. In: Brazilian Symposium on Computers in Education (Simpósio Brasileiro de Informática na Educação-SBIE). [S.1.: s.n.], 2015. v. 26, n. 1, p. 121.

SOUZA, I. M. L. de; RODRIGUES, R. da S.; ANDRADE, W. Explorando robótica com pensamento computacional no ensino médio: Um estudo sobre seus efeitos na educação. In: Brazilian Symposium on Computers in Education (Simpósio Brasileiro de Informática na Educação-SBIE). [S.1.: s.n.], 2016. v. 27, n. 1, p. 490.

VIEIRA, A.; PASSOS, O.; BARRETO, R. Um relato de experiência do uso da técnica computação desplugada. Anais do XXI WEI, p. 670-679, 2013.

WING, J. M. Computational thinking. Communications of the ACM, v. 49, n. 3, p. 33-35, 2006.

ZANETTI, H.; BORGES, M.; RICARTE, I. Pensamento computacional no ensino de programação: Uma revisão sistemática da literatura brasileira. In: Brazilian Symposium 
on Computers in Education (Simpósio Brasileiro de Informática na Educação-SBIE). [S.1.: s.n.], 2016. v. 27, n. 1, p. 21. 